\title{
Human papillomavirus infection and associated lesions in the cervix, anal canal, and mouth of HIV-uninfected women with different HIV risk status in Barcelona
}

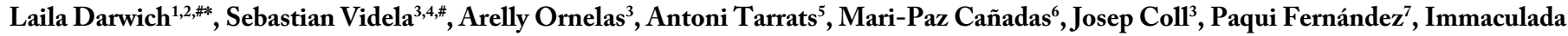 \\ Rivas $^{8}$, Carmen Alcalde ${ }^{3,9}$, Bonaventura Clotet ${ }^{3,9,10}$ and Guillem Sirera ${ }^{3,9}$ \\ ${ }^{1}$ Department of Sanitat i Anatomia Animals, Veterinary School, Cerdanyola del Vallès, 08193, UAB, Catalonia, Spain \\ ${ }^{2}$ Centre en Recerca en Sanitat Animal (CReSA-IRTA), Cerdanyola del Vallès, 08193, Campus UAB, Catalonia, Spain \\ ${ }^{3}$ Lluita Contra La SIDA Foundation. University Hospital Germans Trias i Pujol, Badalona, 08916, Universitat Autònoma de Barcelona (UAB), Catalonia, Spain \\ ${ }^{4}$ Department of Experimental and Health Sciences, Faculty of Health and Life Sciences, Universitat Pompeu Fabra, Barcelona, Catalonia, Spain. \\ ${ }^{5}$ Department of Gynecology and Obstetrics, University Hospital Germans Trias i Pujol, Badalona, 08916, UAB, Catalonia, Spain \\ ${ }^{6}$ Labco-General Lab, Barcelona, 08029, Spain \\ 'PASSIR ("Customer Service in Sexual and Reproductive Health"), Mataró, Catalonia, Spain \\ ${ }^{8}$ Delta Centre, Badalona, Catalonia, Spain \\ ${ }^{9}$ HIV Clinical Unit, Department of Medicine, University Hospital Germans Trias i Pujol, Badalona, 08916, UAB, Catalonia, Spain \\ ${ }^{10}$ Retrovirology Laboratory IrsiCaixa Foundation, University Hospital Germans Trias i Pujol, Badalona, 08916, UAB, Catalonia, Spain \\ \#Both authors have contributed equally to this study
}

\begin{abstract}
Objective: Data on human papillomavirus (HPV) infections and their related lesions appearing simultaneously at different anatomical sites are scarce. Our aim was to provide information about HPV infection and lesions of the cervix, anal canal, and mouth of women at different risk conditions to be infected by HIV.

Methods: Cross-sectional study. Detection and genotyping of HPV infections were done by multiplex PCR, and the cytology by Papanicolaou test. HPV Prevalence and associated risk factors were estimated.

Results: The study included 108 women (44 at risk and 64 with no risk of HIV-infection). The highest prevalence of HPV infection was found in the anal canal (26\% at HIV-risk versus $28 \%$ with no risk group, $\mathrm{p}=0.79)$, followed by the cervix ( $14 \%$ versus $28 \%, \mathrm{p}=0.07)$ and the oral cavity $(7 \%$ versus $9 \%, \mathrm{p}=0.64)$. HPV16 was the most prevalent type in cervix (5.6\%) and anus (8.6\%) for both groups but HPV52 (4.5\%) was the most frequent type in the mouth of the at-risk group. Interestingly, women from the at HIV-risk group presented higher prevalence of anal infection involving a wider range of different high-risk oncogenic HPV-types. Concomitant HPV infections with HPV16, 18 and other oncogenic types (HPV56, 59) were found in 3.1\% of women (all three areas) and 7.1\% (cervix and anal canal). As regards HPV-related lesions, no statistical differences were observed between groups, although women at HIV-risk had higher proportion of anal abnormalities (18.4\% versus $8.2 \%, \mathrm{p}=0.19)$. Finally, cervical HPV-infection was significantly associated with presence of cervical or anal condylomata and alcohol consumption and anal HPV-infection with smoking and RAI.
\end{abstract}

Conclusion: Both women at risk of HIV-infection or with anogenital warts presented a wider range of HPV-oncogenic types in the anal canal, resulting good candidates for HPV anal screening and monitoring programs.

\section{Introduction}

Persistent human papillomavirus (HPV) infection can progress to cervical cancer after several asymptomatic years. The HPV types that infect the anogenital tract are classed as low-risk (LR), mainly HPV-6 and HPV-11, which are associated with anogenital condylomata [1-3], and high-risk (HR), mainly HPV-16 and HPV-18, which are associated with high-grade squamous intraepithelial lesions and squamous anogenital cancers [4-10].

Most recent research has focused on women and is restricted to the cervix, although the increase in HPV-related malignancy at other sites such as the anus, mouth, oropharynx, vulva, vagina, penis, and scrotum has expanded research on HPV [11-14]. However, data on HPV at extra-genital sites are rare and limited to at-risk groups, such as men who have sex with men [15], transplant recipients [16], sex workers [17] and HIV-infected individuals [18-22].

Studies on the prevalence of concomitant HPV infection and the associated lesions at various anatomical sites in general population

Correspondence to: Laila Darwich, DVM, PhD, Associate Professor, School $\mathrm{f}$ Veterinary Medicine, Department of Sanitat i Anatomia Animals, Universitat Autònoma de Barcelona (UAB), 08193, Spain, Tel: +34935811046; Fax: +34935813297; E-mail: Laila.Darwich@uab.cat

Key words: HPV prevalence, predictive factors, cervix, anus, mouth, women, HIV-risk Received: August 20, 2017; Accepted: September 11, 2017; Published: September 14, 2017 
Darwich L (2017) Human papillomavirus infection and associated lesions in the cervix, anal canal, and mouth of HIV-uninfected women with different HIV risk status in Barcelona

including women with a high risk to be infected with HIV are limited $[23,24]$ and uncommon in Europe. Albeit the benefit of HPV vaccination in women before the initiation of their sexual life seems unquestionable, its benefit on other areas HPV-infected remains debated. Given the importance of future cancer prevention strategies worldwide, full characterization of the natural history of HPV at sites where HPV causes cancer should be a priority even in minority populations with especial feature.

This cross-sectional study provides data about the prevalence of HPV, type distribution, and concomitant infection and pathology in the cervix, anal canal, and mouth in a group of women in Barcelona with different HIV-risk behaviors.

\section{Patients and methods}

\section{Study design and population}

Cross sectional study of women (general population) recruited from various health centers: the HPV-Unit from Department of Gynecology and Obstetrics (one morning per week attend HIV-infected women) and the medical school of the University Hospital Germans Trias i Pujol of Badalona, Catalonia, Spain; a women's health center called PASSIR (Atenció a la Salut Sexual i Reproductiva Maresme: “Customer Service in Sexual and Reproductive Health”, Mataró, Catalonia, Spain); and a Local Institute for Personal Services for people with addictions (Delta Centre, Badalona, Catalonia, Spain).

Consecutive visits of women aged $\geq 18$ years were informed about the study and invited to participate. Those who agreed gave their written informed consent, attended an interview, and completed a self-administered questionnaire. They then underwent a clinical examination (systematic visual inspection) of the 3 sites and a digital rectal examination to assess HPV-related pathology. Samples from the 3 areas were collected to detect HPV infection. The sample from the cervix and anal canal was also used for cytology (Papanicolaou test). A blood sample was obtained from all women to rule out HIV infection.

\section{Ethics}

The study was reviewed and approved by the local ethics committee and performed according to the stipulations of the Declaration of Helsinki. Data confidentiality was guaranteed according to the Spanish law on protection of personal data (LOPD 15/1999).

\section{Questionnaire}

The participants completed a detailed ad hoc self-administered questionnaire on their sociodemographic factors, sex practices, health status (including cervical-anal-oral disease and history of sexually transmitted infections), and drug consumption (tobacco, alcohol, intravenous drugs). Data were entered in an electronic medical file with other data (date of birth, date of the visit, and HPV genotyping results for the 3 sites). A health professional (not their primary caregiver) was present to resolve doubts during completion.

\section{Sample collection}

Capillary blood from the fingertip was obtain to perform a rapid test to assess HIV infection. HIV infection was evaluated using the commercial Determine ${ }^{\mathrm{Tx}}$ HIV-1/2 Ag/Ab Combo (Alere Healthcare, S.L.U. Barcelona, Spain). In case of a positive result, this was confirmed by Western Blot and enzyme immunoassay EIA/ELISA. HIV viral load was assessed using Nuclisens (detection limit 80 copies $/ \mathrm{mL}$; bioMérieux, Inc., Durham, North Carolina, USA).
Cervical samples were obtained from the exocervix with an Ayre spatula and from the endocervix with a cotton swab. The spatula and cotton swab were placed into $20 \mathrm{~mL}$ of PreservCyt/ThinPrep Pap test solution (Cytyc Iberia SL, Barcelona, Spain) and shaken for 30 seconds. The solution was stored at $4^{\circ} \mathrm{C}$ until analysis. This sample was used for cytology and DNA extraction. Anal canal and oral epithelial cell samples were obtained as previously described [25].

\section{DNA extraction and HPV genotyping}

DNA was extracted from cell suspensions using the QiAMP Viral DNA kit (QIAGEN, Hilden, Germany) and purified by taking an aliquot of $0.3 \mathrm{~mL}$ from the genital samples and $0.5 \mathrm{~mL}$ from the oral samples. All samples were pelleted by centrifugation and digested for 2 hours at $55^{\circ} \mathrm{C}$ with Proteinase $\mathrm{K}$ at a final concentration of $0.5 \mathrm{mg} / \mathrm{mL}$ (Roche Diagnostic S.L.), followed by heat inactivation at $95^{\circ} \mathrm{C}$ for 10 minutes and kept frozen until PCR. HPV was detected and typed using the F-HPV typing ${ }^{\text {ma }}$ kit (Molgentix, Spain) for HPV 6, 11, 16, 18, 31, 33, $35,39,45,51,52,56,58,59$, and 68 as previously described ${ }^{25}$.

\section{Statistical analysis}

\section{Sample Size}

Using regression models, a sample size 150 HIV-negative has an upper $80 \%$ power to detect an R-square of 0.1 to 15 independent variables allocated using F test with a significance level of 5\%, the upper power when HIV status is considered as a covariate in the model.

\section{Definitions}

The HIV risk status was obtained through the questionnaire. High risk was defined as having at least one of the following criteria: commercial sex workers, sex (vaginal and/or anal) with an HIVinfected male partner with detectable viral load at some time and/or people who inject drugs non-sporadically (background or currently).

The patient was considered to have a detectable HPV infection when $\geq 1$ HPV type was detected in a sample. Prevalent HPV infection was defined as detectable HPV infection (i.e. positive for any of the 15 HPV types investigated). No detectable HPV infection was defined as non-detectable HPV infection (i.e. none of the 15 types was detected).

\section{Statistical procedures}

A descriptive analysis was performed for baseline population characteristics. Prevalence with its $95 \%$ confidence intervals (95\%CI) was calculated for a positive diagnosis of cervical, anal, and oral HPV infection (yes/no) and for type-specific HPV infection. A descriptive analysis of HPV distribution and concomitant HPV infection at the 3 sites was performed. Differences between qualitative variables were evaluated using the $\chi^{2}$ test or Fisher exact test. Associated predictive factors (odds ratio [OR] and $95 \% \mathrm{CI}$ ) were analyzed using multivariate logistic regression models. Statistical significance was set at $\mathrm{p}$-value $\leq$ 0.05. Data were analyzed using SPSS version 15.0.

\section{Results}

\section{Baseline characteristics}

Inclusion ran from April 2008 to February 2011. The study population comprised 108 women (Caucasian and HIV-uninfected) but, 44 (41\%) of them were at risk of HIV infection: 39 women were sexually active (vaginal and/or anal sex) with an HIV-infected male partner; 4 women were injection drug users (past and/or currently); and 1 both, had an HIV-infected partner and was an injection drug user. 
Darwich L (2017) Human papillomavirus infection and associated lesions in the cervix, anal canal, and mouth of HIV-uninfected women with different HIV risk status in Barcelona

The general group presented a median of 36 years of age (Table $1)$, but there was a significant difference between the age of women at risk of HIV infection (42 years) and the other women without risk (27 years). The group at risk presented a higher proportion of women consuming intravenous drugs ( $11 \%$ compared to $2 \%$ of sporadic use in the no risk group). On the other hand, this latest group, the no HIV-risk, presented a higher proportion of women consuming alcohol (around $80 \%$ vs $54 \%$, Table 1). There were no differences between groups as regards to the number of lifetime sexual partners, and the frequency of oral sex or receptive anal intercourse practices.

\section{HPV infection at the different anatomical sites}

The prevalence of cervical HPV infection was estimated including all participants, and it was $23 \%$ (25/108, 95\%CI:16-32), mainly caused by a single HPV type. In fact, multiple-type cervical HPV infections represented only $6 \%$ of all cervical infections in these women. The prevalence of cervical HPV infection was $14 \%(6 / 44,95 \%$ CI:6-28) at risk and $28 \%(18 / 64,95 \% \mathrm{CI}: 18-41)$ at non-risk of HIV $(\mathrm{P}=0.07)$.

The prevalence of anal canal HPV infection was estimated in 93 women, and was $27 \%$ (25/93, 95\%CI: 18-37). In addition, the prevalence of multiple-type anal canal HPV infection was slightly higher than in the cervix (8\%). The prevalence of anal HPV infection was $26 \%(11 / 43$, 95\%CI:14-41) at risk and 28\% (14/50, 95\%CI:17-43) at non risk of HIV $(\mathrm{P}=0.79)$

The prevalence of oral HPV infection was estimated including all participants, and it was $8 \%$ of women (9/108, 95\%CI: $4-15)$. No multiple-type oral infections were detected. The prevalence of oral HPV infection was $7 \%(3 / 44,95 \% \mathrm{CI}: 2-20)$ at risk and $9 \%$ (6/64, 95\%CI:4-20) at non risk of $\mathrm{HIV}(\mathrm{P}=0.64)$.

HPV16 was the most prevalent type in the cervix (5.6\%) and the anal canal $(8.6 \%)$ of the general group. Interestingly, women from the at HIV-risk group presented higher prevalence of anal infection involving a wider range of different HPV types (all 15 genotypes) than the group of no risk $(\mathrm{p}<0.05)$ (Figure 1). However, fewer types of HPV were found in the cervix of the HIV at risk group -with only special relevance of HPV59- as compared to the other group, which presented a high diversity of genotypes. None cervical samples tested positive for HPV58 or HPV68 types.

In the oral site, HPV52 was the most frequent type detected (4.5\%) and exclusively found in the HIV at risk group. The oral HPV16 prevalence was $3.1 \%$ and only detected in the group of no HIV-risk (Figure 1).

\section{Concomitant HPV infection of the cervix, anal canal, and mouth}

The overall prevalence of concomitant HPV infection at all 3 sites was $3.1 \%$ (3/98): 1 woman at HIV risk and 2 women at non-HIV risk. Nevertheless, only 1 woman presented a concomitant infection by the same HPV genotype (HPV56) at all 3 sites.

The prevalence of concomitant HPV infection of the cervix and anus was $7.1 \%(7 / 98)$ and of the cervix and mouth was $1 \%(1 / 98)$ in the overall group (sharing HPV59 and HPV18). Only 1 woman (0.9\%) presented concomitant HPV infection of the cervix and mouth with HPV16. The HPV genotypes involved in concomitant HPV infection of the cervix and anal canal are shown in Table 2.

\section{Cytological findings}

The overall prevalence of cervical and anal cytological abnormalities was $21.5 \%(23 / 107)$ and $12.6 \%$ (11/87), respectively. In detail, $9.3 \%$ $(10 / 107)$ of the cervical samples showed an ASCUS, 8.4\% (9/107) LSIL and $3.7 \%(4 / 107)$ HSIL. In the anal canal, lesions were distributed as 9.2\% (8/87) ASCUS and 3.4\% (3/87) LSIL. No lesions were observed in the oral mucosa.

The prevalence of cervical cytological abnormalities (ASCUS, LSIL and HSIL) was $15.9 \%$ (7/44) in HIV-risk group and $25.4 \%(16 / 63)$

Table 1. Baseline characteristics of non-HIV-infected women stratified by risk of HIV infection. *Median [interquartile range]. Yes (\%): Number of patients (percentage based on data available). ${ }^{1}$ Presence of condylomata at inclusion in the cohort. ${ }^{2}$ In the past and/or currently. ${ }^{3}$ Sporadic consumer (once) STI: sexually transmitted infections.

\begin{tabular}{|c|c|c|c|c|}
\hline Characteristics & $\begin{array}{c}\text { Non-HIV-infected } \\
\quad \mathrm{N}=108\end{array}$ & $\begin{array}{c}\text { At risk of HIV } \\
n=44(41 \%)\end{array}$ & $\begin{array}{c}\text { Not at risk of HIV } \\
n=64(59 \%)\end{array}$ & p-value \\
\hline Age* & $36[25-43]$ & $42[39-47]$ & $27[23-36]$ & $<0.001$ \\
\hline $20-29$ years & $38(35)$ & $1(2)$ & $37(58)$ & \\
\hline $30-39$ years & $33(31)$ & $13(30)$ & $20(31)$ & \\
\hline $40-49$ years & $31(29)$ & $26(59)$ & $5(8)$ & \\
\hline $50-59$ years & $5(5)$ & $3(7)$ & $2(3)$ & \\
\hline $60-69$ years & $1(1)$ & $1(2)$ & $0(0)$ & \\
\hline History of STI [Yes (\%)] & $66(61)$ & $28(64)$ & $38(59)$ & 0.838 \\
\hline \multicolumn{5}{|l|}{ History of condylomata at baseline ${ }^{1}$} \\
\hline Cervical $[$ Yes $(\%)]$ & $15(14)$ & $3(7)$ & $12(19)$ & 0.095 \\
\hline Anal $[$ Yes (\%)] & $3(3)$ & $1(2)$ & $2(3)$ & 1.000 \\
\hline Alcohol consumption $^{2}[$ Yes $(\%)]$ & $71(68)$ & $23(54)$ & $48(79)$ & 0.010 \\
\hline Smoker & & & & 0.678 \\
\hline Never [Yes (\%)] & $34(32)$ & $12(27)$ & $22(34)$ & \\
\hline Past [Yes (\%)] & $17(16)$ & $8(18)$ & $9(14)$ & \\
\hline Current [Yes (\%)] & $54(51)$ & $23(52)$ & $31(50)$ & \\
\hline Injection drug user ${ }^{2}[$ Yes (\%)] & $6(6)$ & $5(11)$ & $1^{3}(2)$ & 0.036 \\
\hline Number of lifetime sexual partners: & & & & 0.307 \\
\hline $1-5$ partners $[$ Yes $(\%)]$ & $68(65)$ & $31(71)$ & $37(58)$ & \\
\hline 6-10 partners [Yes $(\%)]$ & $17(16)$ & $5(11)$ & $12(19)$ & \\
\hline $11-20$ partners [Yes $(\%)]$ & $13(13)$ & $3(7)$ & $10(16)$ & \\
\hline$>20$ partners [Yes $(\%)]$ & $6(6)$ & $3(7)$ & $3(5)$ & \\
\hline Oral sex $[$ Yes $(\%)]$ & $100(95)$ & $38(86)$ & $62(98)$ & 0.165 \\
\hline Receptive anal intercourse ${ }^{2}[$ Yes (\%)] & $52(49)$ & $19(44)$ & $33(52)$ & 0.435 \\
\hline
\end{tabular}


Darwich L (2017) Human papillomavirus infection and associated lesions in the cervix, anal canal, and mouth of HIV-uninfected women with different HIV risk status in Barcelona
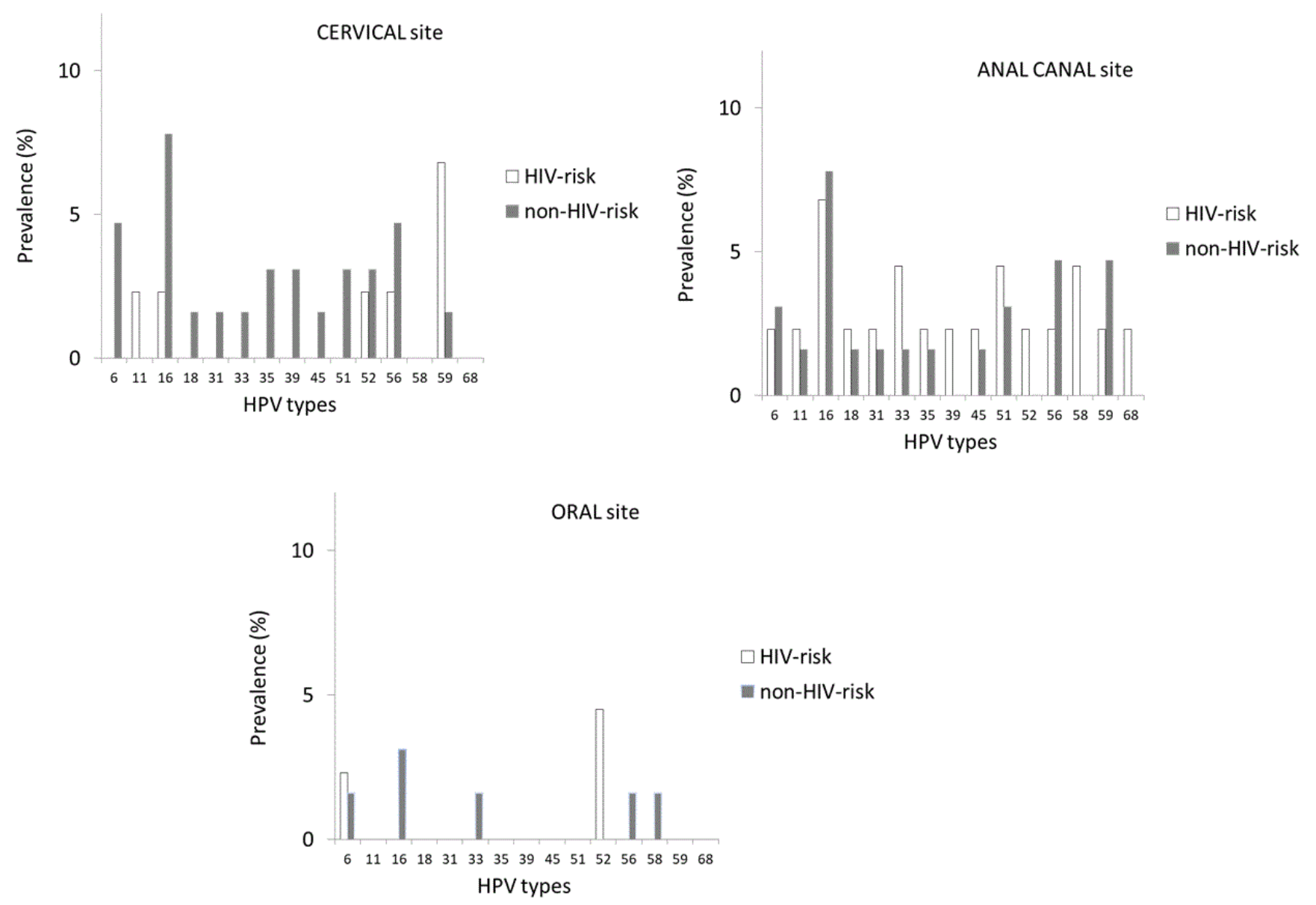

Figure 1. Genotype frequencies of HPV infection at the different anatomical sites and by groups.

Table 3. Predictive factors associated with HPV-infection in the cervix, anal canal, and mouth of non-HIV-infected women. OR: odds ratio. NS: non significant. STI: sexually transmitted infections. RAI: receptive anal intercourse. ${ }^{\mathrm{a} O n l y}$ variables with $\mathrm{p}<0.20$ in the crude analysis were represented in the table. ${ }^{\mathrm{b}}$ The multivariate analyses are adjusted for all the predictors used in the crude analysis. *Included women with history and/or presence of condylomata at baseline. \#In the past and/or currently.

\begin{tabular}{|c|c|c|c|c|}
\hline \multirow[t]{2}{*}{ Cervical HPV infection } & \multicolumn{2}{|c|}{ Crude $^{a}$} & \multicolumn{2}{|c|}{ Adjusted $^{\mathrm{b}}$} \\
\hline & OR $(95 \% C I)$ & p-value & OR $(95 \% C I)$ & p-value \\
\hline Age & $0.93(0.88-0.98)$ & 0.016 & NS & NS \\
\hline History of STI & $2.06(0.74-5.75)$ & 0.166 & NS & NS \\
\hline Cervical condylomata* (yes) & $5.50(1.74-17.34)$ & 0.004 & $5.87(1.64-21.10)$ & 0.007 \\
\hline Anal condylomata* (yes) & $7.55(0.65-87.09)$ & 0.105 & $26.63(1.09-649.76)$ & 0.044 \\
\hline Alcohol consumption $^{\#}$ (yes) & $6.51(1.43-29.71)$ & 0.016 & $14.06(1.70-116.45)$ & 0.014 \\
\hline Smoker $^{\#}($ yes $)$ & $2.26(0.87-5.88)$ & 0.093 & NS & NS \\
\hline $\begin{array}{l}\text { Number of lifetime sexual partners } \\
\qquad>5\end{array}$ & $2.33(0.92-5.93)$ & 0.075 & NS & NS \\
\hline RAI (yes) & $2.24(1.52-11.78)$ & 0.006 & NS & NS \\
\hline \multirow{2}{*}{ Anal HPV infection } & \multicolumn{2}{|c|}{ Crude $^{a}$} & \multicolumn{2}{|c|}{ Adjusted $^{\mathrm{b}}$} \\
\hline & OR $(95 \% C I)$ & p-value & OR $(95 \% C I)$ & p-value \\
\hline Age & $0.94(0.89-0.99)$ & 0.022 & NS & NS \\
\hline Smoker ${ }^{\#}$ (yes) & $7.59(2.34-24.59)$ & 0.001 & $7.92(2.37-26.45)$ & 0.001 \\
\hline Injection drug user ${ }^{\#}$ & $3.15(0.59-16.86)$ & 0.180 & NS & NS \\
\hline $\begin{array}{l}\text { Number of lifetime sexual partners } \\
>5\end{array}$ & $2.55(0.98-6.67)$ & 0.056 & NS & NS \\
\hline RAI (yes) & $2.88(1.09-7.62)$ & 0.033 & $3.09(1.08-8.88)$ & 0.036 \\
\hline \multirow[t]{2}{*}{ Oral HPV infection } & \multicolumn{2}{|c|}{ Crude $^{a}$} & \multicolumn{2}{|c|}{ Adjusted $^{\mathrm{b}}$} \\
\hline & OR $(95 \% C I)$ & p-value & OR $(95 \% C I)$ & p-value \\
\hline Age & $0.95(0.88-1.03)$ & 0.196 & NS & NS \\
\hline
\end{tabular}



status in Barcelona

in non-HIV risk group ( $\mathrm{p}=0.34)$ : ASCUS 9.1\% (4/44) vs 9.5\% (6/63), $\mathrm{p}=1.0$; LSIL $4.5 \%(2 / 44)$ vs $11.1 \%(7 / 63), \mathrm{p}=0.30$; and HSIL $2.3 \%$ $(1 / 44)$ vs $4.8 \%(3 / 63), p=0.64$. Women at HIV risk presented a greater prevalence of anal canal cytological abnormalities (ASCUS, LSIL and HSIL) than the group of non-HIV risk [18.4\% (7/38) versus $8.2 \% 4 / 49)$, $\mathrm{p}=0.19$ ]: ASCUS $13.2 \%(5 / 38)$ vs $6.1 \%(3 / 49), \mathrm{p}=0.26$, LSIL $5.3 \%(2 / 38)$ vs $2.0 \%(1 / 49), \mathrm{p}=0.41$.

Only 2 women of the study presented concomitant lesions in the cervix and anal canal: a woman at HIV risk (ASCUS and ASCUS, respectively), and another woman at non-HIV risk (ASCUS and L-SIL, respectively).

\section{Predictive factors related to HPV infection at the different sites}

The risk of cervical HPV infection was significantly increased by the presence of cervical condylomata $(\mathrm{OR}=5.87,95 \% \mathrm{CI}$ : 1.64-21.1), anal condylomata $(\mathrm{OR}=26.63,95 \% \mathrm{CI}: 1.1-650)$ and alcohol consumption (OR=14.06, 95\%CI: 1.7-116) (Table 3). By contrast, anal HPV infection was associated with smoking $(\mathrm{OR}=7.92,95 \% \mathrm{CI}$ : 2.37-26.45) and RAI $(\mathrm{OR}=3.09,95 \% \mathrm{CI}: 1.08-8.88)$. No risk factors were associated with oral HPV infection (Table 3).

\section{Discussion}

In the present study data on HPV infections and related lesions at different anatomical sites are reported in women at different HIVrisk conditions. Although HPV16 was the most prevalent type in the cervix and the anal canal regardless the risk condition, women at risk of HIV-infection presented higher prevalence of anal infection involving a wider range of different HPV types and a higher proportion of anal cytological abnormalities than the group of no HIV-risk. Sexual behaviour is a key factor in the prevalence of HPV-related diseases. However, in our study there were no statistical differences between groups in the number of sexual lifetime partners or the sexual practices (oral sex and RAI) to explain this differential distribution of the HPV types. However, the study could found differences between the average ages of the groups. As expected, age (older age) was a protective factor for both cervical anal canal HPV infection, and a tendency was found for oral site. In fact, the prevalence of cervical HPV infection goes down with the age $e^{4}$ The difference of age between groups could explain because HIV-risk group (15 years older) presented lower percentage of HPV infection in the cervix ( $14 \%$ vs $28 \%)$. In this line, changes in sexual behaviour are related with an increment of age.
In contrast, the highest HPV prevalence was detected in the anal canal (26\% HIV-risk group, 28\% non HIV-risk group) as compared to cervix and mouth. From another point of view, the prevalence of HPV infection in anal canal was similar between younger $(28 \%)$ and 15 years older (26\%) women, despite age was a protective factor. These results agree with those of studies from the USA, where the greatest burden of anal infection is attributed to a higher incidence and persistence of HPV infection in the anus than at other anatomical sites [19]. Taken together, these data reinforce the role of the anus as a reservoir and source of cervical and oral HPV infection [24].

The prevalence of HPV-related lesions in cervix (21.5\%) was greater than in anal canal (13\%). Although no statistical differences were observed between groups as regards cervical lesions, the no HIV-risk group seemed to have more proportion of lesions $(15.9 \%$ vs $25.4 \%$ ). However, anal HPV-related lesions in the HIV-risk group were greater than the group of women with no HIV-risk $(18,4 \%$ and $8.2 \%$, respectively), although again this difference was not statistically significant. RAI could not explain this result, given that this sexual practice was lower in this group of women on HIV-risk. Likewise, the number of lifetime sexual partners was slightly lower in this group. But, taking into account the results obtained in HPV infection and in HPV-related lesions in cervix and anal canal, these could also suggest that natural history of HPV infection in anal canal differs from cervical infection.

Risk analysis for the detection of anal HPV has been reported mainly in at-risk groups, such as men who have sex with men [26-28] and HIV-infected men [18,20,29]. Risk factors include HIV infection, low CD4 counts, anal condylomata, and history of RAI. Less is known about the risk factors for anal HPV infection in women (with or without HIV infection), although scarce data from the USA indicate that risk factors are similar to those found in men [24,30]. Sexual behavior, i.e., the proportion of women having oral sex and of RAI may have an effect in the prevalence of HPV. In a recent publication conducted in individuals from the general population with genital condylomata, extragenital HPV was common in patients with genital condylomata [31]. In fact, we found that cervical HPV infection was associated with the presence of cervical or anal condylomata and alcohol consumption in our HIV-uninfected women; anal HPV infection was associated with smoking and RAI. A recent study also found that anal sex was associated with persistence of HPV16 in the anus in heterosexual women [32].

Table 2. Concomitant HPV infection in cervix and anal canal [n (\%)].

\begin{tabular}{|c|c|c|c|c|c|c|c|c|c|c|c|c|c|c|c|c|}
\hline & \multirow{2}{*}{$\begin{array}{l}\text { HPV } \\
\text { types }\end{array}$} & & \multicolumn{14}{|c|}{ ANAL CANAL } \\
\hline & & 16 & 18 & 31 & 33 & 35 & 39 & 45 & 51 & 52 & 56 & 58 & 59 & 68 & 6 & 11 \\
\hline \multirow{15}{*}{ 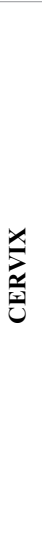 } & 16 & $3(2.8 \%)$ & 0 & 0 & 0 & 0 & 0 & 0 & 0 & 0 & $1(0.9 \%)$ & $1(0.9 \%)$ & 0 & 0 & 0 & 0 \\
\hline & 18 & $1(0.9 \%)$ & 0 & 0 & 0 & 0 & 0 & 0 & $1(0.9 \%)$ & 0 & $1(0.9 \%)$ & 0 & $1(0.9 \%)$ & 0 & 0 & 0 \\
\hline & 31 & 0 & 0 & 0 & 0 & 0 & 0 & 0 & 0 & 0 & 0 & 0 & 0 & 0 & 0 & 0 \\
\hline & 33 & 0 & 0 & 0 & $1(0.9 \%)$ & 0 & 0 & 0 & 0 & 0 & 0 & 0 & $1(0.9 \%)$ & 0 & $1(0.9 \%)$ & 0 \\
\hline & 35 & $1(0.9 \%)$ & 0 & 0 & 0 & 0 & 0 & 0 & 0 & 0 & 0 & 0 & 0 & 0 & 0 & 0 \\
\hline & 39 & 0 & 0 & 0 & 0 & 0 & 0 & 0 & 0 & 0 & $1(0.9 \%)$ & 0 & 0 & 0 & 0 & 0 \\
\hline & 45 & 0 & 0 & 0 & $1(0.9 \%)$ & 0 & 0 & 0 & 0 & 0 & 0 & 0 & $1(0.9 \%)$ & 0 & $1(0.9 \%)$ & 0 \\
\hline & 51 & $1(0.9 \%)$ & 0 & 0 & 0 & 0 & 0 & 0 & $1(0.9 \%)$ & 0 & $1(0.9 \%)$ & 0 & $1(0.9 \%)$ & 0 & 0 & 0 \\
\hline & 52 & 0 & 0 & 0 & 0 & 0 & 0 & 0 & 0 & 0 & 0 & 0 & 0 & 0 & 0 & 0 \\
\hline & 56 & 0 & 0 & 0 & 0 & 0 & 0 & 0 & 0 & 0 & 0 & 0 & 0 & 0 & 0 & 0 \\
\hline & 58 & 0 & 0 & 0 & 0 & 0 & 0 & 0 & 0 & 0 & 0 & 0 & 0 & 0 & 0 & 0 \\
\hline & 59 & $2(1.9 \%)$ & 0 & 0 & 0 & 0 & 0 & 0 & $1(0.9 \%)$ & 0 & $1(0.9 \%)$ & 0 & $1(0.9 \%)$ & 0 & 0 & 0 \\
\hline & 68 & 0 & 0 & 0 & 0 & 0 & 0 & 0 & 0 & 0 & 0 & 0 & 0 & 0 & 0 & 0 \\
\hline & 6 & 0 & 0 & 0 & 0 & 0 & 0 & 0 & 0 & 0 & 0 & 0 & 0 & 0 & 0 & 0 \\
\hline & 11 & 0 & 0 & 0 & 0 & 0 & 0 & 0 & 0 & 0 & 0 & 0 & 0 & 0 & 0 & $1(0.9 \%)$ \\
\hline
\end{tabular}


Darwich L (2017) Human papillomavirus infection and associated lesions in the cervix, anal canal, and mouth of HIV-uninfected women with different HIV risk status in Barcelona

Our study is subject to a series of limitations. First, the crosssectional design could lead to underestimation or overestimation of prevalence at the different sites. Sample size could also lead us to underestimate or overestimate prevalence. Women in this study could be susceptible to HPV infection, i.e., although we aimed to include women from the general population, the women who attended our HIV Unit may have been more likely to acquire HPV infections. Consequently, prevalence may have been overestimated. Besides, the population analyzed, from a single geographical site, could lead us to underestimate or overestimate the generalizability of the results beyond the population and conditions studied.

In summary, women at risk of HIV-infection or with anogenital warts had more probability to be infected in the anal canal and cervix by several HPV high-risk oncogenic types not covered by the current vaccines. Thus, they are good candidates for HPV screening and monitoring for HPV-related diseases in the cervix and anus.

\section{Acknowledgements}

The authors would like to thank the following people: Ms Roser Escrig (Lluita Contra La SIDA Foundation) for monitoring; Ms Ines Fernández and Ms Anna Chamorro (Lluita Contra La SIDA Foundation) for their help in sample management. We are especially grateful to the study participants.

\section{Potential conflicts of interest}

S.V. has received honoraria for collaborating with Laboratorios Dr Esteve on work not related to human papillomavirus/HIV. B.C. has received honoraria for speaking and participating in advisory boards from Abbott, Bristol-Myers Squibb, Boehringer-Ingelheim, Gilead Sciences, GlaxoSmithKline, Pfizer, Merck, Janssen-Tibotec, and Siemens. The remaining authors have no conflict of interest.

\section{Fundings}

This work was supported by grants from Red de Investigación en SIDA (RIS), ART AIDS Foundation, Gilead Sciences, Obra Social Caixa Sabadell, and Gala contra la SIDA-Barcelona 2011, 2012, and 2013.

\section{Contributions}

L. Darwich and S. Videla wrote the manuscript. B. Clotet, G. Sirera and S. Videla designed and wrote the study protocol; P. Fernández, I. Rivas, G. Sirera and A. Tarrats informed non-HIV-infected women about the study; A. Tarrats and G. Sirera visited and interviewed the patients; C. Alcalde, J. Coll, G. Sirera and A. Tarrats collected cervical, anal, and oral specimens; J. Coll carried out the patient coordination; M.P. Cañadas and L. Darwich performed human papillomavirus detection and genotyping with multiplex polymerase chain reaction; A. Ornelas and S. Videla processed the data and performed the statistical analysis; All the authors have read and approved the final manuscript.

\section{References}

1. Dupin N (2004) Genital warts. Clin Dermatol 22: 481-486. [Crossref]

2. Chiao EY, Giordano TP, Palefsky JM, Tyring S, El Serag H (2006) Screening HIVinfected individuals for anal cancer precursor lesions: a systematic review. Clin Infect Dis 43: 223-233. [Crossref]

3. Darwich L, Cañadas MP, Videla S (2012) Condylomata, cytological abnormalities and human papillomavirus infection in the anal canal in HIV-infected men. HIV Med 13: 549-557.

4. Bosch FX, Manos MM, Muñoz N (1995) Prevalence of human papillomavirus in cervical cancer: a worldwide perspective. International biological study on cervical (IBSCC) Study Group. J Nat Cancer Inst 87: 796-802.
5. Walboomers JM, Jacobs MV, Manos MM, Bosch FX, Kummer JA, et al. (1999) Human papillomavirus is a necessary cause of invasive cervical cancer worldwide. $J$ Pathol 189: 12-19. [Crossref]

6. Clifford GM, Smith JS, Plummer M, Muñoz N, Franceschi S (2003) Human papillomavirus types in invasive cervical cancer worldwide: a meta-analysis. $\mathrm{Br} J$ Cancer 88: 63-73. [Crossref]

7. Muñoz N, Bosch X, De SanJosé S (2003) Epidemiologic classification of human papillomavirus types associated with cervical cancer. N Engl J Med 3248: 518-527.

8. Castellsagué X, Díaz M, de Sanjosé S (2006) Worldwide human papillomavirus etiology of cervical adenocarcinoma and its cofactors: Implications for screening and prevention. J Natl Cancer Inst 98: 292-293.

9. Trottier H, Franco EL (2006) The epidemiology of genital human papillomavirus infection. Vaccine 24 Suppl 1: S1-15. [Crossref]

10. Darwich L, Videla S, Cañadas MP (2013) Distribution of human papillomavirus genotypes in anal cytological and histological specimens from HIV-infected men who have sex with men and men who have sex with women. Dis Colon Rectum 56: 1043-1052.

11. De Vuyst H, Clifford GM, Nascimento MC (2009) Prevalence and type distribution of human papillomavirus in carcinoma and intraepithelial neoplasia of the vulva, vagina and anus: a meta-analysis. Int J Cancer 124: 1626-1636.

12. Stanley M (2010) Pathology and epidemiology of HPV infection in females. Gynecol Oncol 117: S5-10. [Crossref]

13. Chaturvedi AK, Anderson WF, Lortet-Tieulent J, Curado MP, Ferlay J, et al. (2013) Worldwide trends in incidence rates for oral cavity and oropharyngeal cancers. $J$ Clin Oncol 31: 4550-4559. [Crossref]

14. Giuliano AR, Nyitray AG, Kreimer AR (2015) EUROGIN 2014 roadmap: Differences in human papillomavirus infection natural history, transmission and human papillomavirus-related cancer incidence by gender and anatomic site of infection. Int $J$ Cancer 136: 2752-2760.

15. Parisi SG, Cruciani M, Scaggiante R (2011) Anal and oral human papillomavirus (HPV) infection in HIV-infected subjects in northern Italy: a longitudinal cohort study among men who have sex with men. BMC Infect Dis 11: 150.

16. Grulich AE, van Leeuwen MT, Falster MO (2007) Incidence of cancers in people with HIV/AIDS compared with immunosuppressed transplant recipients: a meta-analysis. Lancet 370: 59-67.

17. Cañadas MP, Bosch FX, Junquera ML, Ejarque M, Font R, et al. (2004) Concordance of prevalence of human papillomavirus DNA in anogenital and oral infections in a high-risk population. J Clin Microbiol 42: 1330-1332. [Crossref]

18. Palefsky JM, Holly EA, Ralston ML (2001) Prevalence and risk factors for anal human papillomavirus infection in human immunodeficiency virus (HIV)-positive and highrisk HIV-negative women. J Infect Dis 183: 383-391.

19. Beachler DC, D'Souza G, Sugar EA, Xiao W, Gillison ML (2013) Natural history of anal vs oral HPV infection in HIV-infected men and women. J Infect Dis 208: 330-339. [Crossref]

20. Videla S, Darwich L, Cañadas MP (2013) Natural history of human papillomavirus infections involving anal, penile, and oral sites among HIV-positive men. Sex Transm Dis 40: 3-10

21. Darwich L, Cañadas MP, Videla S (2013) Prevalence, clearance, and incidence of human papillomavirus type-specific infection at the anal and penile site of HIVinfected men. Sex Transm Dis 40: 611-618.

22. Darwich L, Cañadas MP, Videla S (2014) Oral human papillomavirus type-specific infection in HIV-infected men: a prospective cohort study among men who have sex with men and heterosexual men. Clin Microbiol Infect 20: 585-589.

23. Crawford R, Grignon AL, Kitson S, Winder DM, Ball SL, et al. (2011) High prevalence of HPV in non-cervical sites of women with abnormal cervical cytology. BMC Cancer 11: 473. [Crossref]

24. Hessol NA, Holly EA, Efird JT, Minkoff H, Weber KM, et al. (2013) Concomitant ana and cervical human papillomavirusV infections and intraepithelial neoplasia in HIVinfected and uninfected women. AIDS 27: 1743-1751. [Crossref]

25. Videla S, Darwich L, Cañadas MP (2009) Epidemiological data of different human papillomavirus genotypes in cervical specimens of HIV-1-infected women without history of cervical pathology. J Acquir Immune Defic Syndr 50: 168-75.

26. Melbye M, Smith E, Wohlfahrt J (1996) Anal and cervical abnormality in women prediction by human papillomavirus tests. Int J Cancer 68: 559-564. 
Darwich L (2017) Human papillomavirus infection and associated lesions in the cervix, anal canal, and mouth of HIV-uninfected women with different HIV risk status in Barcelona

27. Cañadas MP, Videla S, Darwich L (2010) Human papillomavirus HPV-16, 18, 52 and 58 integration in cervical cells of HIV-1-infected women. J Clin Virol 48: 198-201.

28. Critchlow CW, Hawes SE, Kuypers JM, Goldbaum GM, Holmes KK, et al. (1998) Effect of HIV infection on the natural history of anal human papillomavirus infection. AIDS 12: 1177-1184. [Crossref]

29. Holly EA, Ralston ML, Darragh TM, Greenblatt RM, Jay N, et al. (2001) Prevalence and risk factors for anal squamous intraepithelial lesions in women. $J$ Natl Cancer Inst 93: 843-849. [Crossref]
30. Hessol NA, Holly EA, Efird JT, Minkoff H, Schowalter K, et al. (2009) Anal intraepithelial neoplasia in a multisite study of HIV-infected and high-risk HIVuninfected women. AIDS 23: 59-70. [Crossref]

31. Kofoed K, Sand C, Forslund O, Madsen K (2014) Prevalence of human papillomavirus in anal and oral sites among patients with genital warts. Acta Derm Venereol 94: 207 211. [Crossref]

32. Moscicki AB, Ma Y, Farhat S, et al. Natural history of anal human papillomavirus infection in heterosexual women and risks associated with persistence. Clin Infect Dis 58: 804-811.

Copyright: (C2017 Darwich L. This is an open-access article distributed under the terms of the Creative Commons Attribution License, which permits unrestricted use, distribution, and reproduction in any medium, provided the original author and source are credited. 